\title{
The Population Predicting Based on the Curve Fitting Least Square Method
}

\author{
Lili $\mathrm{HE}^{1, \mathrm{a}}$,Zhao $\mathrm{JIN}^{2^{*}, \mathrm{~b}}$ \\ ${ }^{1}$ College of Information Science and Electronic Technology JIA MU SI University,China \\ ${ }^{2} \mathrm{JIA} \mathrm{MU} \mathrm{SI} \mathrm{Aviation} \mathrm{Forest} \mathrm{Fire,China}$ \\ ajmshelili@163.com, ${ }^{b} 12316036 @ q q . c o m$
}

Keywords: Curve Fitting; Malthusian model; prediction model

\begin{abstract}
In various scientific experiments, experimenter always get some discrete data, to estimate the function with the original rules from these data, interpolation is a method for dealing with this kind of problem.But when the discrete data is too much,it can be caused serious distortion which is called Runge phenomenon at the interval endpoints.To avoid, the Curve Fitting Least Square method is a kind of optimal solution. The Curve Fitting Least Square method is used to be dealing with structure prediction model etc.In this paper, Malthusian model is the method of population predicting.In Malthusian model,the unknown variable is ascertained by the Curve Fitting Least Square method.
\end{abstract}

\section{Introduction}

In the scientific experiments or the statistical research,always get some discrete data from experiment or sampling,recorded as $\left(x_{i}, y_{i}\right)(i=0, \cdots n)$.Try to structure the function $p(x)$ by these discrete data to replace the original function $f(x)$.The feature of commonly used interpolation method is the fitting curve crossing those interpolation points,such as Largange,Newton,Hermite and Cubic Spline etc.Although some data may be somewhat distorted by the experiment ,or defective data, interpolation method can take those errors to $p(x)$, and given the inevitable adverse effects. The Curve Fitting Least Square method is not pass by every ineterpolation point,which is pass through those point by fitting thought,to get the change trend of curve and isolate causal,and reflect the objective fact.

\section{The principle of the Curve Fitting Least Square method}

If the experiment dataset is $\left(x_{i}, y_{i}\right)(i=0, \cdots n)$, and the fitting function is $p(x)$,then the deviation which is maked from every interpolation point is $\delta_{i}=\left|p\left(x_{i}\right)-y_{i}\right|(i=0, \cdots n) \cdot p(x)$ can express the original function $f(x)$, and make $\delta_{i}$ minimum. Usually use these standards as a measure of the deviation:

$$
\left\|\delta_{i}\right\|_{1}=\sum_{i=0}^{n}\left|\delta_{i}\right|, \quad\left\|\delta_{i}\right\|_{2}=\left(\sum_{i=0}^{n} \delta_{i}^{2}\right)^{\frac{1}{2}}, \quad\left\|\delta_{i}\right\|_{\infty}=\max _{0 \leq i \leq n}\left|\delta_{i}\right|
$$

The Curve Fitting Least Square method use minimum 2-norm.

More specifically, for a group of experiment data $\left(x_{i}, y_{i}\right)(i=0, \cdots n)$, try to struct a function as $p(x)=a_{0} \varphi_{0}(x)+a_{1} \varphi_{1}(x)+\cdots a_{m} \varphi_{m}(x)=\sum_{j=0}^{m} a_{j} \varphi_{j}(x), \varphi(x)$ is the basis function in it, When $\left\|\delta_{i}\right\|_{2}^{2}=\sum_{i=0}^{n} \delta_{i}^{2}=\sum_{i=0}^{n}\left(p\left(x_{i}\right)-y_{i}\right)^{2}$ is least, $\left(a_{0}, a_{1}, \cdots a_{m}\right)^{T}$ is the solution. 
Multi-variable function is $F\left(a_{0}, a_{1}, \cdots a_{n}\right)=\left\|\delta_{i}\right\|_{2}^{2^{2}}=\sum_{i=0}^{n}\left(\sum_{j=0}^{m} a_{j} \varphi_{j}\left(x_{i}\right)-y_{i}\right)$, let $\frac{\partial F}{\partial a_{k}}=0$ $(k=0,1 \cdots, m)$, then $\frac{\partial F}{\partial a_{k}}=\sum_{i=0}^{n}\left(2\left(\sum_{j=0}^{m} a_{j} \varphi_{j}\left(x_{i}\right)-y_{i}\right) \varphi_{k}\left(x_{i}\right)\right)=2 \sum_{j=0}^{m} \sum_{i=0}^{n} a_{j} \varphi_{j}\left(x_{i}\right) \varphi_{k}\left(x_{i}\right)-2 \sum_{i=0}^{n} y_{i} \varphi_{k}\left(x_{i}\right)=0$

This is the final result which the above fomular after deducing.

$$
\sum_{j=0}^{m} \sum_{i=0}^{n} a_{j} \varphi_{j}\left(x_{i}\right) \varphi_{k}\left(x_{i}\right)=\sum_{i=0}^{n} y_{i} \varphi_{k}\left(x_{i}\right)(k=0,1 \cdots, m) .
$$

$\operatorname{Let}\left(\varphi_{j}, \varphi_{k}\right)=\sum_{i=0}^{n} \varphi_{j}\left(x_{i}\right) \varphi_{k}\left(x_{i}\right), \quad\left(f, \varphi_{k}\right)=\sum_{i=0}^{n} y_{i} \varphi_{k}\left(x_{i}\right)=d_{k}$, then this fomula is $\sum_{j=0}^{m}\left(\varphi_{k}, \varphi_{j}\right) a_{j}=d_{k}$ $(k=0,1 \cdots, m)$.And turn it to the matrix:

$$
\left(\begin{array}{cccc}
\left(\varphi_{0}, \varphi_{0}\right) & \left(\varphi_{0}, \varphi_{1}\right) & \ldots & \left(\varphi_{0}, \varphi_{m}\right) \\
\left(\varphi_{1}, \varphi_{0}\right) & \left(\varphi_{1}, \varphi_{1}\right) & \ldots & \left(\varphi_{1}, \varphi_{m}\right) \\
\vdots & \vdots & \vdots & \vdots \\
\left(\varphi_{m}, \varphi_{0}\right) & \left(\varphi_{m}, \varphi_{1}\right) & \ldots & \left(\varphi_{m}, \varphi_{m}\right)
\end{array}\right)\left(\begin{array}{c}
a_{0} \\
a_{1} \\
\vdots \\
a_{m}
\end{array}\right)=\left(\begin{array}{c}
d_{0} \\
d_{1} \\
\vdots \\
d_{m}
\end{array}\right) .
$$

This is a linear system of equations which coefficient is $a_{j}(k=0,1 \cdots, m)$, and it also called canonical equation.For the vector $\left(\varphi_{0}, \varphi_{1}, \cdots \varphi_{m}\right)$ is linearly independent,then this linear system of equations' s determinant is not zero,so this linear system of equations have have only one solution.

\section{Algorithm design}

Curve Fitting Least Square method consists of two parts,first,get the augmented matrix of the linear system of equations's determinant.Second,get the solution of the linear system of equations. Use the Gauss method to get the solution.The specific algorithm is in the figures 1 .

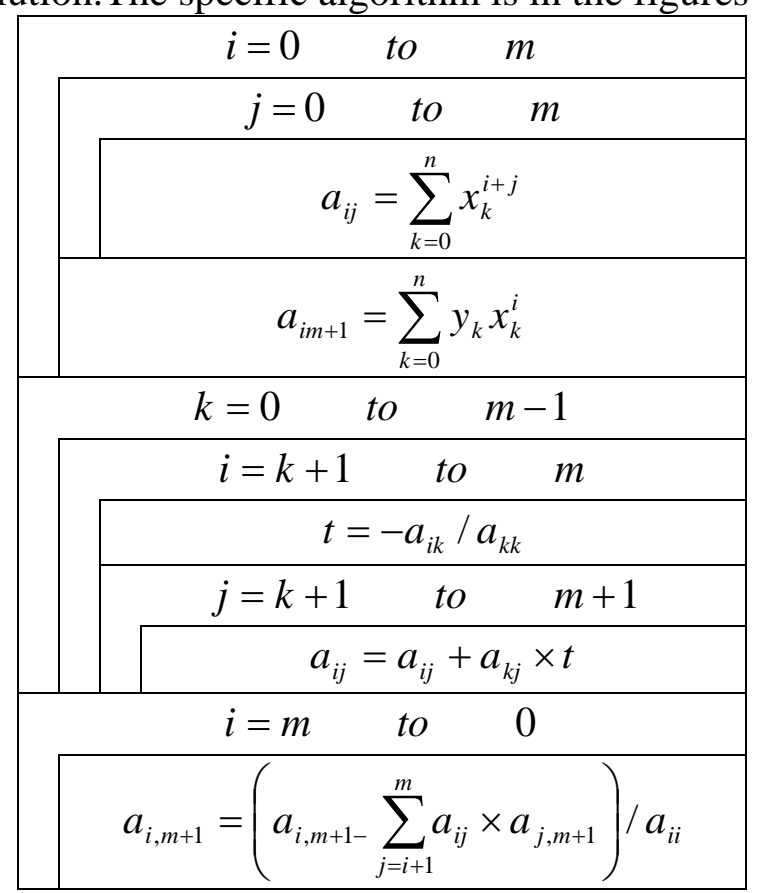

Fig.1: the algorithm description

In figure 1-1, $n$ is the high limit of those interpolation points' $s$ subscript, $m$ is the highest power of empiric formulas, and $X 、 Y$ are the Domain and range of interpolation points. 


\section{Malthusian model}

Malthusian model is a model about population growth.Let total population is $N(x)$, for the theory of population as Malthusian model, use the exponential function as $N(x)=e^{a_{0}+a_{1} x}$, and in this expression $x$ is the year.And make the $\log$ of this expression,we can get the equation as $y(x)=\ln N(x)=a_{0}+a_{1} x$.In the equation, $a_{0}$ and $a_{1}$ are unknown,they need fitting from those ineterpolation points,for the empiric formulas $y(x)$ is linear,and the quantity of ineterpolation points is more than $2(\geq 2)$,so use the Curve Fitting Least Square method to struct fitting function is appropriate.

For example,Chease population is known int the past ten years as table 1.

Table 1: Chease population in last ten years

\begin{tabular}{ccccccccccc}
\hline \multirow{2}{*}{ Year } & 200 & 200 & 200 & 200 & 200 & 200 & 201 & 201 & 201 & 201 \\
& 4 & 5 & 6 & 7 & 8 & 9 & 0 & 1 & 2 & 3 \\
\hline Quant & & & & & & & & & & \\
ity(hu & 12. & 13. & 13. & 13. & 13. & 13. & 13. & 13. & 13. & 13. \\
ndred & 998 & 075 & 144 & 212 & 280 & 347 & 410 & 473 & 540 & 607 \\
millio & 8 & 6 & 8 & 9 & 2 & 4 & 0 & 5 & 4 & 2
\end{tabular}

n)

From the Curve Fitting Least Square method,get the linear system of equations about $a_{0}$ and $a_{1}$ :

$\left(\begin{array}{cc}10 & 20085 \\ 20085 & 4.0340800\end{array}\right)\left(\begin{array}{l}a_{0} \\ a_{1}\end{array}\right)=\left(\begin{array}{l}25.8834 \\ 51987.3\end{array}\right)$

After solving,the fitting function is $y(x)=-7.50297+0.0050243 \times x$.And put the 2014 to the Malthusian model,then the forecast is $e^{-7.50297+0.0050243 \times 2014} \approx 13.6806$ hundred million.It can be expressed in figure. 2 and figure 3,and in the figure. 2 it is the rule in the domain,and in the figure 3it is the rule in the past ten years.It is log function rule in all the domain,but in the past ten years,it is resembling a straight line.From the figure 3,it can be found that fitting curve is not pass on every point which is pass through.

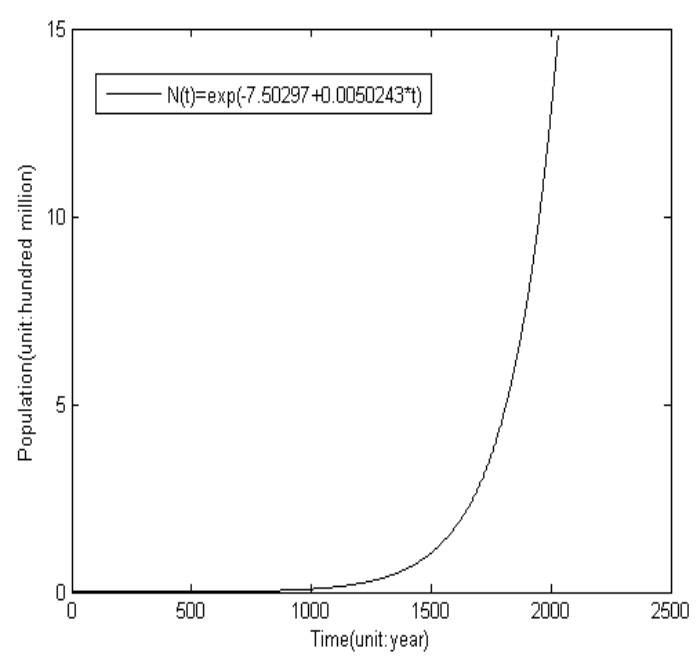

Fig.2: fitting function in the domain 


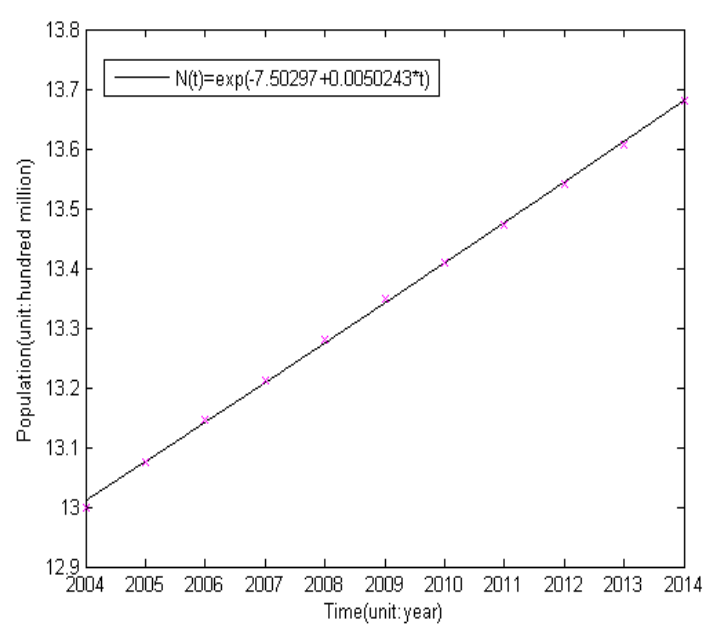

Fig.3: fitting function in the past ten years

\section{Conclusion}

The Malthusian model which is used to be forcasting population is mature and widely used.It would be assistant decision-making about decisions of a nation or area scientifically and reasonably.And the Curve Fitting Least Square method is excellent in the prediction field.Both of these method could reach the changes of the population veritably.

\section{Acknowledgement}

This work is supported by the JIA MU SI University science and technology research project(No.L2013-075)

*Corresponding author.

\section{References}

[1] YU Hongjun.Two Geometric Methods of Curve-fitting.Science Technology and Engineering.Vol.12 N0.16 Jun.2012

[2] Fu Yanru.Rearch on Actual Measurement on Matlab Curve Fitting.Journal of Jinlin Normal University.No.2.May.2010

[3] Yang Lijian,Sun Xiao,Gao Songwei.Application of curve fitting in signal reconstruction of magnetic flux leakage inner detection for pipeline.Journal of Shenyang University of Technology.Vol.34 No.6 Nov.2012

[4] Bian Huanqing,Xia letian.The Application of Gray Markov Model in Population Forcasting.Mathematics in Practice and Theory.Vol42 No.7.Apr.2012

[5] He Lili.Least Squares Algorithm Desgin and C++ Realization of Curve Fitting.Journal of Hunan Post and Telecommunication College.Vol.13 No.1.Mar.2014

[6] Ren Qiang,Hou Dadao.Stochastic Model for Population Forecast:Based on Leslie Matrix and ARMA Model.Population Research.Vol35 No.2.March 2011

[7] Hu Qingwan.Curve Fitting by Curve Fitting Toolbox of Matlab.Computer Knowledge and Technology.Vol.6 No.21.July 2010

[8] Liu Nian,Du Qiuxia,Liu Gang,Yu Peiyi.Research and Application of Curve-Fitting Based on Function Combination.Computer and Information Technology.Vol.21 No.4.Aug.2013

[9] Xu Linzhou,Zhang Xinhua,Song Yuan.Bearings-only Target Para meter Estim ation A Lgorithm for Stationary Single-Station based on Curve-fit.Fire Control and Comm and Control.Vol35.Aug 2010

[10]Zeng Jinfang,Teng Zhaosheng,Application of information entropy in curve fitting recognition.Journal of Electronic Measurement and Instrument.Vol26 No.2.Febr 2012 